\title{
Phytotherapeutika wirken anders - und oft auch besser
}

\section{Warum Phytotherapie konventioneller Pharmakotherapie ưberlegen sein kann}

\section{Cäcilia Brendieck-Worm}

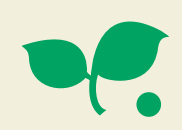

\section{Zusammenfassung}

Phytotherapeutika zeigen seltener unerwünschte Arzneimittelwirkungen und sind dadurch meist besser verträglich als die konventionelle Pharmakotherapie. Da Phytotherapeutika jedoch meist ein Gemisch vieler Substanzen sind, sind oft wirksamkeitsbestimmende Inhaltsstoffe im Einzelnen nicht darstellbar - ein Umstand, der dazu führt, dass viele Phytotherapeutika mit jahrhundertelangen Anwendungserfahrungen vom Markt genommen werden müssen. Der Artikel beschreibt die Unterschiede zwischen Phyto- und konventioneller Therapie und zeigt Anwendungsgebiete auf, bei denen Phytotherapie überlegen ist.

Haustiere entwickeln zunehmend Gesundheitsstörungen, die den „Wohlstandserkrankungen“ von uns Menschen ähneln. Trotz enormen Zuwachses an diagnostischen und therapeutischen Möglichkeiten wird eine Heilung der Tiere oft nicht erreicht. Vielfach wird durch unangepasste Pharmakotherapie zusätzlich Schaden und Leid verursacht. Aufgrund der z.T. spärlichen Datenlage in der Pharmakotherapie der Tiere und den daraus entstehenden unkalkulierbaren Risiken ist generell zu erwägen, ob Phytotherapeutika den synthetischen Pharmaka vorgezogen werden können und sollten.

\section{Fallstricke der konventionellen Therapie Pharmakokinetik und -dynamik}

Konventionelle Pharmakotherapie erweist sich beim Tier in vielen Fällen deshalb als problematisch, da der größte Teil der Pharmaka für die Anwendung beim Menschen entwickelt wurde. Tierartliche Unterschiede in Pharmakokinetik und -dynamik finden kaum Berücksichtigung. Diese Unter-

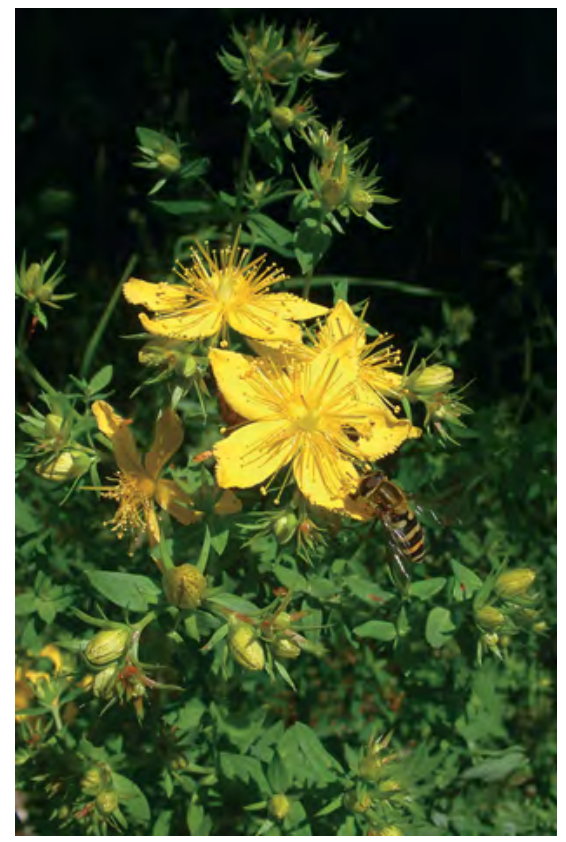

Abb. 1 Johanniskraut - bei Depressionen ein potentes Phytotherapeutikum (৫ Ferdinand Worm).

schiede verursachen jedoch bei gleicher Dosierung sehr unterschiedliche Wirkqualitäten [10].

Durch unterschiedliche Verteilung im Organismus, Bioverfügbarkeit, Halbwertszeit und auch durch Kumulation aufgrund fehlender oder ineffizienter Detoxifikationsmöglichkeiten (Glukuronidierungsschwäche der Katze, Acetylierungsschwäche des Hundes etc.) kann die Anwendung ineffektiv, sinnlos oder sogar gefährlich sein [10]. Es besteht meist keine lineare Beziehung zwischen Dosis und Körpergewicht, was Dosisangaben in $\mathrm{mg} / \mathrm{kg}$ problematisch macht [9].

Ein weiteres Problem ist, dass bei Tierarzneimitteln viele pharmakokinetische Angaben vom gesunden, erwachsenen Tier stammen [10]. Für Jungtiere, alte und kranke Tiere fehlt häufig eine fundierte Datenbasis. Und auch hier sind i.d.R. nur die bedeutsamen Tierarten wie Hunde, seltener auch Katzen untersucht.

Im Gegensatz dazu gibt es meist umfassende Angaben zu Pharmakokinetik und -dynamik für lebensmittelliefernde Tiere. Sie haben bei diesen Tierarten zu einer deutlichen Einschränkung der Pharmakotherapie geführt (Rückstandsproblematik, Wartezeiten, Verbraucherschutz).

\section{latrogene Einflüsse und Chronifizierung}

Da therapeutische Bemühungen häufig nur dazu verhelfen sollen, den gesundheitsschädlichen Lebensstil bzw. die nicht artgerechte Nutz- und Massentierhaltung aufrechterhalten zu können, nehmen iatrogene Schäden zu.

So ist der zunehmend praktizierte Langzeit- und Dauereinsatz steroidaler und nichtsteroidaler Antiphlogistika, z.B. bei chronischen Gelenks- und Darmentzündungen, kritisch zu sehen, da er die Situation zementiert und degenerative Prozesse verstärkt.

\section{Dauer- und Komedikation}

Dauermedikation führt zur Gewöhnung. Das bedeutet Wirkungsverlust und macht Dosissteigerungen nötig. Gleichzeitig steigt die Gefahr unerwünschter Arzneiwirkungen. Insbesondere hepato- und nephrotoxische sowie immunsystemschädigende Effekte der Medikation führen zu einer Verschlechterung des Gesundheitszustands durch die Medikation und machen Komedikationen notwendig.

Besonders davon betroffen ist der multimorbide oder geriatrische Patient, der ohnehin in seinen Ausleitungs- und Detoxifikationsleistungen geschwächt ist. So bedürften beispielsweise durch die Hauptmedikation entstandene Blutgerinnungsund Blutbildungsstörungen, Spontanfrakturen oder unkontrollierbare Infektionen 
durch bakterielle Resistenzen (v.a. bei Hospitalisierung) ihrerseits einer Therapie. Wechselwirkungen der eingesetzten Medikamente werden unüberschaubar, führen zu weiterem Leid bis hin zu lebensbedrohlichen Störungen und Tod.

Auch bei Tieren werden zunehmend invasive Eingriffe als „Reparaturmaßnahmen“ vorgenommen, wie Endoprothesen, Implantate, Transplantationen etc. Dies macht immunsuppressive Therapie und antibiotische „Abdeckung“ notwendig. Wundheilungsstörungen und Resistenzbildung bei Hospitalismuskeimen (z.B. Multiresistenter-Staphylococcus-A, kurz MRSA in der Humanmedizin) sind die Folge, erhöhen die Kosten, verlängern das Leiden und führen nicht selten zu Folgeschäden bis hin zum Tod.

Schlechte Erfahrungen mit Nebenwirkungen synthetischer Arzneimittel haben bewirkt, dass heute wieder vermehrt darüber nachgedacht wird, Phytotherapeutika den Vorzug zu geben. So hat die Contergan-Katastrophe der 1950er-Jahre viele Menschen sensibilisiert, zumindest während der Schwangerschaft zurückhaltend mit dem Einsatz synthetischer Arzneimittel zu sein. Hier ließen sich noch viele Beispiele anführen.

\section{Wissenschaftliche Anerken- nung der Phytotherapie}

Es besteht die Gefahr, dass wissenschaftliche Medizin bei der Nutzung von Heilpflanzen an ihrem eigenen Anspruch der Messbarkeit und Objektivierbarkeit bzw. an deren finanziellen Folgen scheitert, wenn historische Quellen der Heilpflanzenkunde und die heutige volks- und naturheilkundliche Praxis mit ihrem riesigen Erfahrungsschatz diskriminiert werden [2, 4].

Behördlicherseits wird nicht berücksichtigt, dass diese z.T. sehr umfangreichen Anwendungserfahrungen und die Ausrichtung auf übergreifende Wirksamkeit, sprich Aktivierung der umfassenden Selbstheilungsmöglichkeiten des Organismus, eine Therapie mit Heilpflanzen bereits als unbedenklich ausgewiesen haben. Dies gilt insbesondere für Heilpflanzen mit Breitbandwirkstoffen.

Bedenklich ist auch, dass „im Dienste der Wissenschaft" jeder nichtstoffliche Anteil der Therapie eliminiert wird [2].
Das Ergebnis, der randomisierte, placebokontrollierte Doppelblindversuch, ist trotz seiner schon vor über 30 Jahren nachgewiesenen Unzulänglichkeit in der Medizin immer noch Goldstandard heutiger Wissenschaft und Grundlage evidenzbasierter Medizin [6].

\section{Wirkungs- und \\ Unbedenklichkeitsnachweis}

Vonseiten der rationalen Phytotherapie wird betont, dass Phytotherapie ohne Einschränkung auf der naturwissenschaftlichen Medizin basiert. Sie hat also keine eigenen Theorien oder Lehren bezüglich des menschlichen/tierischen Körpers, seiner Funktionsweisen, der Entstehung und Erkennung von Krankheiten und der Art und Weise, wie Wirkstoffe Körperfunktionen beeinflussen. Es besteht ein Zusammenhang von Dosis und Wirkung. Pflanzliche Arzneimittel werden folgerichtig denselben pharmakologischen, toxikologischen und klinischen Prüfverfahren unterzogen wie chemisch definierte Arzneimittel [7].

Arzneimittelrechtlich gilt die Heilpflanze bzw. die aus ihr zubereitete Arznei als ein Wirkstoff. Tatsächlich ist sie jedoch ein Vielstoffgemisch, dessen einzelne Komponenten zu einem Gesamteffekt führen. Die einzelnen zum Gesamteffekt führenden Komponenten sind mit den Methoden der pharmakologischen, toxikologischen und klinischen Forschung zum großen Teil charakterisierbar und objektivierbar [7].

Welchen enormen zeitlichen und finanziellen Umfang aber eine „Volldeklaration“ nach sich ziehen würde, lässt sich gerade für die Drogen ohne eindeutig erkennbaren Wirkstoff wie z.B. Johanniskraut (Abb. 1) oder Weidenrinde kaum abschätzen, da für ein Verständnis der Gesamtwirkung auch die Aufklärung synergistischer und antagonistischer Interaktionen innerhalb des Vielstoffgemischs notwendig wäre. Aus wissenschaftlicher Sicht sind solche Untersuchungen hochinteressant. Sie unter dem Aspekt des Wirkungs- und Unbedenklichkeitsnachweises zur Zulassungsbedingung für pflanzliche Arzneimittel zu machen, wäre fatal [8].

Eine Reihe üblicher Beurteilungskriterien lässt sich auf Phytotherapeutika nur bedingt anwenden. So kann die Bioverfügbarkeit eines Stoffgemischs nur anhand ausgewählter Inhaltsstoffe charakterisiert werden, nicht aber für das Vielstoffgemisch. In-vitro-Untersuchungen sind nicht zielführend bei pflanzlichen Wirkstoffen, die in vivo wirksamkeitsbestimmenden metabolischen Prozessen unterliegen. Nicht nur sog. Prodrugs (z.B. das Salicin der Weidenrinde), sondern alle Heilpflanzen mit Breitenwirkungen sind fast ausschließlich durch In-vivo-Studien überprüfbar [7].

In der Wissenschaft verschiebt sich zurzeit - bedingt durch die immer wieder offenbar werdenden Sicherheitsrisiken von chemisch definierten Arzneimitteln der Schwerpunkt der Diskussion von der Wirksamkeit eines Präparats auf Aspekte der Sicherheit und Unbedenklichkeit [8]. Dies betrifft in der Tiermedizin insbesondere die Pharmakotherapie lebensmittelliefernder Tiere (Verbraucherschutz).

Dies müsste sich nach allem bisher Gesagten als Vorteil für die Phytotherapie auswirken. Tatsächlich wird aber der Nachweis von Wirksamkeit und Unbedenklichkeit von Arzneimitteln zur Einschätzung des Nutzen-Risiko-Verhältnisses durch die Zulassungs- und Überwachungsbehörden dabei zum Politikum und erweist sich als Konkurrenzvorteil für chemisch definierte Stoffe, da die Hersteller von Phytotherapeutika als Vielstoffgemischen oder gar Kombinationen von Vielstoffgemischen den Aufwand zur Lieferung dieser Nachweise kaum mehr finanzieren können. Diese „Schwachstelle“ der Phytotherapie wird von der Konkurrenz gezielt genutzt. Schon wenige, z.T. wegen hochgradiger Mängel in der Dokumentation kaum beurteilbare „Schadensmeldungen“ führen bei Phytotherapeutika zur Notwendigkeit kostspieliger zusätzlicher Studien zum Nachweis der Unbedenklichkeit oder gar zum sofortigen Verbot der Anwendung. So geschehen bei Kava-Kava, einem seit Jahrhunderten erfolgreich eingesetzten Anxiolytikum, dem man Fälle von schwerwiegender Leberschädigung zuschrieb, wodurch der Markt wieder frei wurde für die Benzodiazepine, deren Hepatotoxizität deutlich höher liegt [9]. 


\section{UAWs - Diskrepanzen in der Beurteilung}

Heilpflanzen sind nicht per se nebenwirkungsarm oder -frei. Man denke nur an die Giftigkeit großer Heilpflanzen wie Tollkirsche oder Fingerhut. Heute gebräuchliche Phytotherapeutika zeichnen sich jedoch durch sehr gute Verträglichkeit aus.

Doch wenn es um unerwünschte Arzneimittelwirkungen (UAW) geht, wird die Diskrepanz in der Beurteilung synthetischer Pharmaka und Phytotherapeutika durch die Überwachungsbehörden offensichtlich: Während man bei synthetischen Pharmaka UAW aufgrund eines anerkannten Nutzens in Kauf nimmt, werden Phytotherapeutika schon beim Verdacht von Nebenwirkungen reglementiert, da ihnen ein Nutzen abgesprochen oder dieser angesichts des potenziellen Risikos für zu gering gehalten wird.

Phototoxizität und Allergisierungspotenzial sind dabei bevorzugtes Thema. Bei synthetischen Pharmaka wohlbekannt und toleriert, werden sie bei Phytotherapeutika schon bei Verdacht oder theoretischer Möglichkeit problematisiert, ohne dass sie beim Patienten zu beobachten gewesen wären [2]. Hier wird prinzipiell sinnvolle und notwendige Pharmakovigilanz zur Diskriminierung von Konkurrenten missbraucht [14]. Dies soll anhand einiger Beispiele verdeutlicht werden:

Johanniskraut So wurde in diversen Untersuchungen bestätigt, dass therapeutische Dosierungen von Johanniskraut keinen messbaren Einfluss auf die Photosensibilität der Haut haben. Viele synthetische Antidepressiva, wie Amitriptylin, Doxepin oder Clomipramin besitzen allerdings nachweislich photosensibilisierende Eigenschaften - schon in therapeutischer Dosis [17]. Trotzdem wird JohanniskrautExtrakt gern als Musterfall für phototoxische Präparate herausgestellt [13].

Ginkgo Der Verdacht, UAW auszulösen, wird auch für den zur Therapie des demenziellen Syndroms eingesetzten Ginkgo immer wieder erhoben. Anhand der Meldefälle unerwünschter Arzneiwirkungen zeigte sich für den alternativ verordneten Acetylcholinesterasehemmer $(\mathrm{ACH})$ ein 85-fach höheres Risiko für UAW als für den Ginkgo-Extrakt, bei 5-fach höheren Behandlungskosten unter den $\mathrm{ACH}$ [14]. Aufgrund von Einzelfallmeldungen wurde der Verdacht geäußert, Ginkgo würde die Wirkung gerinnungshemmender Arzneistoffe wie Acetylsalicylsäure und von Antikoagulanzien vom CumarinTyp verstärken und das Blutungsrisiko erhöhen. Studien konnten jedoch den Verdacht eines erhöhten Blutungsrisikos unter Komedikation mit Ginkgo widerlegen $[11,12]$.

Beinwell und Huflattich Besonders schwerwiegend ist der Verdacht auf Mutagenität und Kanzerogenität, wie er v. a. bei pyrrolizidinalkaloidhaltigen Pflanzen wie Beinwell und Huflattich erhoben wird. Bei der Beurteilung derartiger Risiken wird von wissenschaftlicher Seite die DosisWirkungs-Beziehung ignoriert und die Möglichkeit absolut gesetzt, dass bereits ein einziges Molekül krankheitsauslösend sein kann. Es wird z.T. über Monate und Jahre mit Dosierungen weit über dem therapeutisch Notwendigen „getestet“, wobei den Versuchstieren diese hohen Dosen nur noch über die Zwangsernährung via Magensonde zugeführt werden können. Den Ergebnissen derart „inquisitorisch“ anmutender Versuche stehen z.B. für Beinwell positive Anwendungserfahrungen aus mehr als 2000 Jahren gegenüber.

\section{Breitbandwirkstoff versus Einzelsubstanz}

Heilpflanzen enthalten ein komplexes Gemisch unterschiedlicher (Sekundär-)Stoffe, die sich im Laufe der Evolution entwickelt haben, um sich vor Fressfeinden, Bakterien, Viren, Pilzen, UV-Strahlung oder Konkurrenten etc. zu schützen $[16,17]$. Dabei sind Wirkstoffe mit hoher Selektivität entstanden (z.B. Atropin, Digitoxin), die wegen ihrer starken Wirkung heute als exakt dosierbare Einzelsubstanzen Verwendung finden, per definitionem aber keine Phytotherapeutika mehr sind.

Gleichzeitig haben sich in $90 \%$ der Heilpflanzen sog. „Breitbandwirkstoffe“ entwickelt, in denen sich keine einzelnen die Wirkung verursachenden Inhaltsstoffe ausmachen lassen. Es wirkt das Stoffgemisch als Ganzes. Die Breitbandwirkstoffe sind in einem seit Millionen Jahren andauernden Prozess in ihrer Wirkung auf biologische Systeme optimiert und haben einen auf übergreifende Wirksamkeit ausgerichteten Ansatz [16]. Sie richten sich z.B. gegen wichtige Zellstrukturen, wie etwa biologische Zellmembranen, die sowohl bei Fressfeinden als auch bei Bakterien und Pilzen vorkommen. Beispiele für derartige Breitbandwirkstoffe sind etwa Senföle, Iridoide, Polyene und Sesquiterpenlactone.

Auf der anderen Seite haben sich bei Mensch und Tier im Zuge der gemeinsamen Evolution mit den Pflanzen Regulative entwickelt, mit denen sie den „feindlichen" Reaktionen der von ihnen aufgenommenen Pflanzen entgegenwirken: vermehrte Sekretion der Verdauungsdrüsen, forcierte Harnausscheidung, Stoffwechselanregung, Durchblutungs-, Diaphorese-, Phagozytosesteigerung usw. Diese macht sich die Phytotherapie zunutze.

Viele Heilpflanzen bergen viele unterschiedliche Wirkungen in sich. So wirken z.B. Ätherischöl-Drogen wie der Thymian sowohl sekretionsfördernd und spasmolytisch als auch antimikrobiell. Da diese Wirkungen den gesamten Organismus betreffen, wird Thymian sowohl bei MagenDarm-Störungen als auch bei Atemwegserkrankungen erfolgreich eingesetzt.

Traditionell werden Heilpflanzen zudem kombiniert, da man so additive und synergistische Effekte erreichen kann (z.B. die Kombinationen von primär ausleitenden, antiphlogistischen und immunmodulierenden Heilpflanzen bei Rheuma).

Im Gegensatz dazu wird mit synthetischen Pharmaka i.d.R. versucht, möglichst spezialisiert einzugreifen. Dabei werden Wirkungen vielfach nur dadurch erreicht, indem körpereigene Regulative unterdrückt oder ausgeschaltet werden. Hierdurch entstehen weitere Abhängigkeiten von Pharmaka. So entstehen durch den Einsatz von steroidalen und nichtsteroidalen Antiphlogistika bei chronischen Entzündungen häufig therapiebedürftige Blutgerinnungsstörungen, Magen-DarmGeschwüre und Osteoporose.

\section{Wo kann Phytotherapie eingesetzt werden? Befindlichkeitsstörungen}

Phytotherapeutika werden v.a. zur Therapie von Befindlichkeitsstörungen eingesetzt. Mit diesem Bereich tut sich die moderne Medizin schwer, da in diesem Stadium der Erkrankung (noch) keine objektivierbaren Befunde zu erheben sind - 


\section{Geschichtlicher Exkurs Phytotherapie}

\section{Pflanzen als Nahrungsgrundlage}

Pflanzen sind seit Jahrmillionen Nahrungsgrundlage der meisten Tiere und Menschen. Zwischen Pflanzen einerseits und Mensch und Tier andererseits hat eine evolutionäre Anpassung stattgefunden [2, 16]. Zudem haben Zuchtauswahl, Anbau-, Zubereitungs- und Konservierungstechniken die Nutzbarkeit der Pflanzen erhöht - eine kulturelle Leistung des Menschen.

Eine besondere Stellung zwischen Nahrungs- und Heilpflanze nehmen die Gewürze ein, deren appetitanregende, verdauungsfördernde, stoffwechselaktivierende und antimikrobielle (konservierende) Eigenschaften von alters her intuitiv genutzt werden. Ihre Wirkprinzipien sind heute Gegenstand wissenschaftlicher Untersuchungen, insbesondere in der Nutztiermedizin.

\section{Pflanzen als Heilmittel}

Tiere unterscheiden zwischen Nahrungspflanzen und Pflanzen, die nur bei bestimmten Befindlichkeitsstörungen und Erkrankungen verzehrt werden. Heilpflanzenkundige früherer Zeiten verweisen auf die Tiere als Quelle ihres Heilpflanzenwissens [1].

Die Anwendung von Pflanzen und deren Zubereitungen zur Heilung oder Linderung von Beschwerden und Krankheiten ist die älteste Therapieform überhaupt. Bereits im Mittelalter haben Menschen begonnen, das Wirkprinzip von Heilpflanzen zu erforschen [16]. Schon Paracelsus war auf der Suche nach ihrer „Quinta essentia“ $[7,8]$.

Als vor mehr als 200 Jahren dem Apotheker Sertürner die Isolierung des Morphins gelang, sah man sich in der Ansicht bestätigt, dass es „den“ wirksamen Stoff in der Pflanze zu finden gelte. Es wurden in der Folgezeit viele potente Wirkstoffe aus Pflanzen isoliert, die noch heute als halb oder rein synthetische Stoffe die Basis der Pharmakotherapie bilden, deren Einsatzmöglichkeiten enorm erweiterten und ihre Effizienz steigerten [8].

Pflanzen dienten als Vorlage für synthetische Modifikationen und Neuschöpfungen. Dabei spielte neben der Verbesserung pharmakologischer Eigenschaften auch der Wunsch nach exklusiver Verfügbarkeit und Patentierbarkeit eine wichtige Rolle [7]. Die chemische Veränderung von pflanzlichen Wirkstoffen führte jedoch schon früh zu unliebsamen Überraschungen. Heroin und LSD verließen einst als vielversprechende Medikamente die Laboratorien und treiben seither in diversen Modifikationen ihr Unwesen in der Welt.

Vonseiten der Wissenschaft war und ist man bestrebt, sich unabhängig zu machen von Naturgegebenheiten und ihren Unwägbarkeiten wie klima- und standortbedingten Schwankungen von Inhaltsstoffen, die dadurch unsichere Reproduzierbarkeit von Extrakten und entsprechende Variabilität bei der Wirksamkeit [8].

Der Wert von Heilpflanzen ohne deutlich erkennbaren und isolierbaren Wirkstoff wurde nicht erkannt, da das Augenmerk auf den Wirkstoff bzw. die Wirkung gerichtet wurde. Dies führte zur Geringschätzung von mehr als $90 \%$ der traditionell genutzten Heilpflanzen, nämlich all jener Drogen, die ihre Wirkung nur als kompositorisches Ganzes entfalten und deshalb bei einer pharmakologischen Überprüfung einzelner Bestandteile zwangsläufig „durchfallen“ - wenn man die klinischen Anwendungserfahrungen unberücksichtigt lässt.

In der Volksmedizin und vom Naturheilkundler werden diese traditionellen Heilpflanzen weiterhin geschätzt.
Phytotherapie heute -

Entwicklung von Spezialextrakten Die phytopharmakologische Forschung der letzten Jahrzehnte bot die Möglichkeiten zur Entwicklung von Spezialextrakten mit eigenem Wirkprofil aus Pflanzen, bei welchen das wirksame Prinzip (nicht nur ein einziger Wirkstoff!) konzentriert wird und gegebenenfalls bedenkliche Inhaltsstoffe reduziert werden können. Das lässt Phytotherapie für die wissenschaftliche Medizin wieder interessant werden. Spezialextrakte sind patentierbar - wegen der immens hohen Kosten für die Entwicklung einer Heilpflanze zum Arzneimittel ist dies eine Grundvoraussetzung für ihre Wirtschaftlichkeit $[7,8]$.

Seit Anfang der 1990er-Jahre haben Johanniskraut-Extrakte in der Therapie der Depression durch ihre gute Wirkung bei deutlich geringeren (Folge-)Kosten und besserer Verträglichkeit als bei chemischen Antidepressiva in der Humanmedizin große Verbreitung erreicht. Ebenso konnte Ginkgo seine gute Wirksamkeit und Unbedenklichkeit bei Hirndurchblutungsstörungen und Demenz unter Beweis stellen. In der Lebertherapie sind Mariendistel-Extrakte Mittel der Wahl.

Die Konkurrenzfähigkeit heutiger, in ihrer Wirksamkeit wissenschaftlich bestätigter Phytotherapeutika mit chemisch definierten Produkten multinationaler Pharma-Riesen bleibt dennoch fraglich. Denn die Finanzkraft dieser Unternehmen ermöglicht ihnen eine globale Einflussnahme durch hoch effiziente Werbung, sowohl auf Laien als auch auf Fachkreise.

Heute ist es ohnehin für die Rehabilitierung vieler pflanzlicher Arzneien zu spät. Sie sind zum größten Teil aufgrund gesetzlicher Regelungen und "gesundheitspolitischer" Entscheidungen (Verlust der Erstattungsfähigkeit durch die gesetzlichen Krankenkassen) vom Markt verschwunden. oder der Therapeut glaubt, auf die Befindlichkeit des Patienten aufgrund der Schwere der Befunde und der deshalb nötigen konventionellen Therapie keine Rücksicht nehmen zu können. Ein Beispiel hierfür und zugleich ein wichtiges Indikationsgebiet für die Phytotherapie sind die Befindensstörungen unter der Behandlung tumoröser Erkrankungen [2]. Das Einbeziehen der Befindlichkeit des Patienten in Diagnose und Therapie gehört unverzichtbar zu ganzheitlicher Medizin.

\section{Chronische Krankheiten}

Gerade Frühformen und Latenzstadien solcher Krankheiten, die zur Chronifizierung neigen, sind ein wichtiges Einsatzgebiet für die Phytotherapie [2]. In vielen Fällen können Phytotherapeutika das Fortschreiten degenerativer Prozesse verhin- dern, verzögern und zu subjektiver und objektiver Besserung führen.

Da erfahrungsgemäß die Gefahr von schädlichen Interaktionen zwischen synthetischen Pharmaka und Phytotherapeutika gering ist, empfiehlt sich etwa bei akuten Schüben von Arthrosen, parallel zur konventionellen Pharmakotherapie mit der Phytotherapie mit Weidenrinde, Weihrauch oder Teufelskralle zu beginnen. Häufig kann so nach wenigen Behandlungstagen das nebenwirkungsreichere synthetische Medikament abgesetzt oder aber mit niedrigerer und damit besser verträglicher Dosierung fortgesetzt werden.

\section{Risikopatienten}

Traditionelle Phytotherapeutika besitzen meist eine große therapeutische Breite. In der Pflanzenheilkunde kann man diesbezüglich auf jahrtausendelange Anwendungserfahrungen zurückgreifen. Dies ist ein wichtiges Kriterium bei der Behandlung von Risikopatienten. Hierzu gehören Neugeborene bzw. Jungtiere, für die es oft keine Daten zur Pharmakokinetik chemisch definierter Pharmaka gibt. Andere Verteilung des Pharmakons durch andere Körperzusammensetzung führt zu Veränderungen bei Wirkungseintritt, -stärke und -dauer. Es kann zu Schäden in unausgereiften Geweben und Organen kommen. Auch die Passage der Blut-Hirn-Schranke ist vielfach noch möglich. Es besteht die Gefahr der Störung der in der Entwicklung befindlichen essenziellen Mikroflora. Durch unausgereifte Metabolisierungsfähigkeiten des Neugeborenen können das Pharmakon und pharmakologisch aktive 
Metabolite kumulieren. Probleme entstehen zudem bei der Dosierbarkeit.

\section{Geriatrie}

Auch alte und vorgeschädigte Tiere sind Risikopatienten. Veränderte Pharmakokinetik, veränderte Metabolisierung durch nachlassende oder gestörte Organleistung und Multimorbidität erhöhen das Risiko einer konventionellen Pharmakotherapie. Besonders Dauer- und Komedikationen sind in ihrer Wirkung nicht mehr überschaubar.

\section{Krankheitsprävention}

Der Phytotherapeut ist mit der von außen durchgeführten Bekämpfung von Krankheitserregern und Noxen und der Unterdrückung der durch sie ausgelösten Symptomatik nicht zufrieden, sondern hat die Aktivierung der Selbstheilungskräfte des Patienten zum Ziel. Deshalb kommt dem präventiven Einsatz von Phytotherapeutika eine große Bedeutung zu. Es sollten belastende Situationen frühzeitig erkannt und bereits bestehende Befindensstörungen ernst genommen werden. Therapeut und Tierbesitzer müssen das Ökosystem, in das jeder Organismus integriert ist, in ihre Beobachtungen mit aufnehmen.

Pflanzliche Immunstimulanzien, z.B. Echinacea, erweisen sich als sinnvoll während nasskalter Witterung, bevor Symptome von Erkältungskrankheiten auftreten. Ebenso vor stressenden Situationen wie Transport, Ausstellungen, neuer Gruppenzusammenstellung etc.

Durchblutungsfördernde und ausleitende Phytotherapeutika wie Brennnessel, Birke oder Löwenzahn beugen vor bei bekannter familiärer oder konstitutioneller Disposition zu Stoffwechselstörungen wie Arteriosklerose, Rheuma, Diabetes oder beim geriatrischen Patienten.

Hepatoprotektiva wie Mariendistel oder Artischocke, die begleitend bei infektiösen Hepatitiden, bei nicht vermeidbarer leberbelastender Medikation und beim geriatrischen Patienten eingesetzt werden, unterstützen u.a. die Regeneration der Leber.
Was gilt es bei der Anwendung zu beachten?

Erfolgreicher Einsatz von Phytotherapeutika setzt die Kenntnis der Inhaltsstoffe, ihrer Wirkungen, der sich daraus ergebenden Indikationsgebiete und zu berücksichtigenden tierartlichen Unterschiede voraus. Der Dosisberechnung ist das metabolische Körpergewicht $\left(\mathrm{KM}_{\mathrm{kg}}{ }^{0,75}\right)$ zugrunde zu legen. Wie auch bei konventionellen Therapeutika sind eine eingehende Anamnese, klinische Untersuchung und Diagnosestellung unabdingbare Voraussetzungen für die richtige Wahl des Phytotherapeutikums.

Das Wirkmaximum wird bei Phytotherapeutika manchmal erst nach 1-2 Wochen erreicht. Akut- und Notfallmedizin sind deshalb nicht ihr Indikationsgebiet. Phytotherapeutika zeigen sich jedoch wegen ihrer Nebenwirkungsarmut in der Langzeit- und Daueranwendung den chemisch definierten Arzneimitteln oft deutlich überlegen.

\section{Fazit}

In vielen Fällen gibt es wichtige Gründe, Phytotherapeutika den chemisch definierten Arzneimitteln vorzuziehen. Wo Therapiefreiheit gegeben ist und Leitlinien sowie Therapie-Empfehlungen als Entscheidungshilfe statt als Diktat verstanden werden, muss eine fundierte Begründung der Vorzüge auch zur Berechtigung der Anwendung führen. Der Therapeut muss sich seiner Verantwortung bei jeglicher Pharmakotherapie bewusst sein. Die Frage: „Ist dieses Arzneimittel sinnvoll und vertretbar?" ist für jeden Patienten individuell zu beantworten, denn dies ergibt sich nicht allein aus den quantitativen Nutzen-Risiko-Berechnungen der Hersteller. Wichtigen Einfluss auf die Entscheidung hat hier das Wissen um Alternativen.

Die Nutzung von Pflanzen in der indigenen Phytotherapie ist Teil eines komplexen kulturellen Ganzen. Der derzeitige drastische Wandel aller Kulturen bedeutet einen dramatischen Verlust an kultureller Vielfalt und Biodiversität und damit auch von über Jahrtausende gewachsenem Heilpflanzenwissen [4]. Das zigtausendfache Screenen von Pflanzen auf der Suche nach neuen Wirkstoffen könnte wahrscheinlich wesentlich effizienter verlaufen, wenn die ethnopharmazeutische Forschung intensiviert würde.

Bewahrung und Erweiterung des phytotherapeutischen Wissens kann das Spektrum therapeutischer Möglichkeiten wesentlich vergrößern.

Das zeigt die Bedeutung von Fort- und Weiterbildung - für denjenigen, der seine therapeutischen Möglichkeiten ausschöpfen möchte, und für all diejenigen, die hier gute Erfahrungen weitergeben können.

\section{() Summary}

\section{Alternative activity of phytothera- peutic agents: Why phytotherapy can supersede conventional pharma- cotherapy}

Phytotherapeutic agents seldom cause undesired side effects and offer better tolerability compared to conventional pharmacotherapy. However, herbal remedies comprise of a mixture of different substances rendering it difficult to determine their individual ingredients. Their acceptance is therefore based on several centuries of application. The article describes the differences between phytotherapy and conventional therapy and highlights the areas where phytotherapy offers better results.

\section{? Key words}

phytotherapy - different substance mixtures - advantages - activity mechanism - risk minimisation

\section{Literatur}

Literatur ist in der Online-Version unter www.thieme-connect.de/ejournals einsehbar.

\section{- Dr. Cäcilia Brendieck-Worm}

Talstraße 59

67700 Niederkirchen

E-Mail: fw@fworm.de

Geb. 1957, 1976-1981 Studium der Veterinärmedizin in Gießen, 1982-1986 Promotion am Institut für Tierzucht und Haustiergenetik der JLU Gießen, seit 1985 Mitglied einer tierärztlichen Praxis für Groß- und Kleintiere in der Pfalz, Zusatzbezeichnung und Weiterbildungsermächtigung Biologische Tiermedizin, Leitung des Arbeitskreises Phytotherapie der GGTM. 